International Journal of Pure and Applied Mathematics

Volume 100 No. 3 2015, 423-433

ISSN: 1311-8080 (printed version); ISSN: 1314-3395 (on-line version)

url: http://www.ijpam.eu

doi: http://dx.doi.org/10.12732/ijpam.v100i3.10

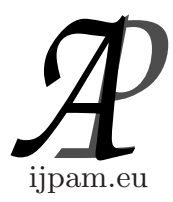

\title{
COMMON FIXED POINT THEOREMS FOR INTEGRAL APPINGS SATISFYING IMPLICIT RELATIONS IN MENGER SPACES
}

\author{
Shin Min Kang ${ }^{1}$, Vishal Gupta ${ }^{2}$, Balbir Singh ${ }^{3}$, Sanjay Kumar ${ }^{4}$ \\ ${ }^{1}$ Department of Mathematics \\ Research Institute of Natural Science \\ Gyeongsang National University \\ Jinju, 660-701, KOREA \\ ${ }^{2,3}$ Department of Mathematics \\ Maharishi Markandeshwar University \\ Mullana, Ambala, Haryana, INDIA \\ ${ }^{4}$ Department of Mathematics \\ Deenbandhu Chhotu Ram University of Science and Technology \\ Murthal, Sonipat, Haryana, INDIA
}

\begin{abstract}
In this paper, we prove a common fixed point theorems using weakly reciprocally continuous, non-compatible self-mappings for integral mappings satisfying some implicit relations in Menger spaces.

AMS Subject Classification: 47H10, 54H25

Key Words: reciprocal continuity, weakly reciprocal continuity, $R$-weakly commuting mappings, $R$-weakly commuting of the type $\left(A_{f}\right)$, of the type $\left(A_{g}\right)$ and of the type $(P)$
\end{abstract}

\section{Introduction and Preliminaries}

The theory of probabilistic metric spaces is an important part of stochastic

Received: December 26, 2014

(c) 2015 Academic Publications, Ltd.

$\S$ Correspondence author url: www.acadpubl.eu 
Analysis, and so it is of interest to develop the fixed point theory in such spaces. The first result from the fixed point theory in probabilistic metric spaces is obtained by Sehgal and Bharucha-Reid [9].

Definition 1.1. (see [8]) A mapping $f: \mathbb{R} \rightarrow \mathbb{R}^{+}$is called distribution function if it is non-decreasing and left-continuous with $\inf \{F(t): t \in \mathbb{R}\}=0$ and $\sup \{F(t): t \in \mathbb{R}\}=1$. We will denote $\mathcal{L}$ by the set of all distribution functions.

Definition 1.2. (see [3]) A probabilistic metric space is a pair $(X, F)$, where $X$ is a nonempty set and $F: X \times X \rightarrow \mathcal{L}$ is a mapping defined by $F(x, y)=F_{x, y}$ satisfying for all $x, y, z \in X$ and $t, s \geq 0$,

(p1) $F_{x, y}(t)=1$ for all $t>0$ if and only if $x=y$,

(p2) $F_{x, y}(0)=0$

(p3) $F_{x, y}(t)=F_{y, x}(t)$,

(p4) If $F_{x, y}(t)=1$ and $F_{y, z}(s)=1$, then $F_{x, z}(t+s)=1$.

Every metric space $(X, d)$ can always be realized as a probabilistic metric space by considering $F: X \times X \rightarrow \mathcal{L}$ defined by $F_{x, y}(t)=H(t-d(x, y))$ for all $x, y \in X$, where $H$ is a specific distribution function (also known as Heaviside function) defined by

$$
\begin{cases}0, & \text { if } t \leq 0, \\ 1, & \text { if } t>0 .\end{cases}
$$

So probabilistic metric spaces offer a wider framework than that of the metric spaces and are general enough to cover even wider statistical situations.

Definition 1.3. (see [8]) A mapping is called a $t$-norm if

(t1) $\Delta(a, 1)=a, \quad \Delta(0,0)=0$,

(t2) $\Delta(a, b)=\Delta(b, a)$,

(t3) $\Delta(c, d) \geq \Delta(a, b)$ for $c \geq a$ and $d \geq b$,

(t4) $\Delta(\Delta(a, b), c)=\Delta(a, \Delta(b, c))$ for all $a, b, c \in[0,1]$.

Example 1.4. The following are the four basic t-norms:

(1) The minimum $t$-norm $\Delta_{M}(a, b)=\min \{a, b\}$;

(2) The product $t$-norm $\Delta_{P}(a, b)=a b$;

(3) The Lukasiewicz $t$-norm $\Delta_{L}(a, b)=\max \{a+b-1,0\}$; 
(4) The weakest $t$-norm (drastic product)

$$
\Delta_{D}(a, b)= \begin{cases}\min \{a, b\}, & \text { if } \max \{a, b\}=1, \\ 0, & \text { otherwise }\end{cases}
$$

In respect of above mention $t$-norms, we have the following ordering:

$$
\Delta_{D}<\Delta_{L}<\Delta_{P}<\Delta_{M} .
$$

Throughout this paper, $\Delta$ stands for an arbitrary continuous $t$-norm.

Definition 1.5. (see [3]) A Menger space is a triplet $(X, F, \Delta)$, where $(X, F)$ is a probabilistic metric space and $\Delta$ is a $t$-norm with the following condition for all $x, y, z \in X$ and $t, s \geq 0$,

$$
\text { (p5) } F_{x, z}(t+s) \geq \Delta\left(F_{x, y}(t), F_{y, z}(s)\right) \text {, }
$$

Definition 1.6. (see [4]) A sequence $\left\{x_{n}\right\}$ in a Menger space $(X, F, \Delta)$ is said to be

(i) convergent at point $x \in X$ if for every $\epsilon>0$ and $\lambda>0$, there exists a positive integer $N_{\epsilon, \lambda}$ such that $F_{x_{n}, p}(\epsilon)>1-\lambda$ for all $n>N_{\epsilon, \lambda}$;

(ii) Cauchy sequence in $X$ if for every $\epsilon>0$ and $\lambda>0$, there exists a positive integer $N_{\epsilon, \lambda}$ such that $F_{x_{n}, x_{m}}(\epsilon)>1-\lambda$ for all $m, n>N_{\epsilon, \lambda}$;

(iii) complete if every Cauchy sequence in $X$ is convergent in $X$.

Definition 1.7. (see [3]) Let $f$ and $g$ be self-mappings of a Menger space $(X, F, \Delta)$. Then $f$ and $g$ are said to be compatible if

$$
\lim _{n \rightarrow \infty} F\left(f g x_{n}, g f x_{n}, t\right)=1
$$

whenever $\left\{x_{n}\right\}$ is a sequence in $X$ such that $\lim _{n \rightarrow \infty} f x_{n}=\lim _{n \rightarrow \infty} g x_{n}=u$ for some $u \in X$ and for all $t>0$.

Definition 1.8. Let $f$ and $g$ be self-mappings of a Menger space $(X, F, \Delta)$. Then $f$ and $g$ are said to be non-compatible if either $\lim _{n \rightarrow \infty} F\left(f g x_{n}, g f x_{n}, t\right)$ is non-existent or

$$
\lim _{n \rightarrow \infty} F\left(f g x_{n}, g f x_{n}, t\right) \neq 1
$$

whenever $\left\{x_{n}\right\}$ is a sequence in $X$ such that $\lim _{n \rightarrow \infty} f x_{n}=\lim _{n \rightarrow \infty} g x_{n}=u$ for some $u \in X$ and for all $t>0$. 
In 2007, Kohli and Vashistha [2] introduced the notions of $R$-weak commutative mappings as follows.

Definition 1.9. Let $f$ and $g$ be self-mappings of a Menger space $(X, F, \Delta)$. Then $f$ and $g$ are said to be

(1) weakly commuting if $F(f g x, g f x, t) \geq F(f x, g x, t)$ for all $x \in X$ and $t>0$

(2) $R$-weakly commuting if there exists $R>0$ such that $F(f g x, g f x, t) \geq$ $F(f x, g x, t / R)$ for all $x \in X$ and $t>0$;

(3) $R$-weakly commuting of the type (i) if there exists $R>0$ such that $F(g f x, f f x, t) \geq F(g x, f x, t / R)$ for all $x \in X$ and $t>0$;

(4) $R$-weakly commuting of the type (ii) if there exists $R>0$ such that $F(f g x, g g x, t) \geq F(f x, g x, t / R)$ for all $x \in X$ and $t>0$;

(5) $R$-weakly commuting of the type (iii) if there exists $R>0$ such that $F(f f x, g g x, t) \geq F(f x, g x, t / R)$ for all $x \in X$ and $t>0$.

In our further discussion, from Imdad and Ali [1], We rename $R$-weakly commuting mappings of the type (i), $R$-weakly commuting mappings of the type (ii) and $R$-weakly commuting mappings of the type (iii) by $R$-weakly commuting mappings of the type $\left(A_{g}\right), R$-weakly commuting mappings of the type $\left(A_{f}\right)$ and $R$-weakly commuting mappings of the type $(P)$, respectively.

Notice that Definition 1.9 was introduced by Imdad and Ali [1] and Pathak et. al. [7] in in a fuzzy metric space and a metric space, respectively.

Clearly, every weakly commuting mappings is $R$-weakly commuting with $R=1$. Moreover, all the notions of $R$-weak commutativity and $R$-weak commutativity of the type $\left(A_{g}\right)$, of the type $\left(A_{f}\right)$ and of the type $(P)$ coincide at coincidence points. Furthermore, all the four notions of $R$-weak commutativity are distinct.

In 1999, Pant [5] introduced a new continuity condition, known as reciprocal continuity as follows.

Definition 1.10. Let $f$ and $g$ be two self-mappings. Then $f$ and $g$ are called reciprocally continuous if $\lim _{n \rightarrow \infty} f g x_{n}=f z$ and $\lim _{n \rightarrow \infty} g f x_{n}=f z$, whenever $\left\{x_{n}\right\}$ is a sequence such that $\lim _{n \rightarrow \infty} f x_{n}=\lim _{n \rightarrow \infty} g x_{n}=z$ for some $z \in X$.

If $f$ and $g$ are both continuous, then they are obviously reciprocally continuous, but the converse is not be true. 
Recently, Pant et al. [6] generalized the notion of reciprocal continuity to weak reciprocal continuity as follows.

Definition 1.11. Let $f$ and $g$ be two self-mappings. Then $f$ and $g$ are called weakly reciprocally continuous if $\lim _{n \rightarrow \infty} f g x_{n}=f z$ or $\lim _{n \rightarrow \infty} g f x_{n}=$ $f z$, whenever $\left\{x_{n}\right\}$ is a sequence such that $\lim _{n \rightarrow \infty} f x_{n}=\lim _{n \rightarrow \infty} g x_{n}=z$ for some $z \in X$.

If $f$ and $g$ are reciprocally continuous, then they are obviously weak reciprocally continuous, but the converse is not true.

\section{Fixed Point Theorems using Implicit Relations}

The Implict Relations $(\Phi)$ : Let $\Phi$ denote the family of functions $\phi:[0,1]^{5} \rightarrow$ $[0,1]$ such that $\phi$ is continuous and $\phi(x, 1,1, x, x)=x$.

These are examples.

(1) $\phi_{1}\left(x_{1}, x_{2}, x_{3}, x_{4}, x_{5}\right)=\min \left\{x_{1}, x_{2}, x_{3}, x_{4}, x_{5}\right\}$.

(2) $\phi_{2}\left(x_{1}, x_{2}, x_{3}, x_{4}, x_{5}\right)=\frac{x_{1}\left(x_{1}+x_{2}+x_{3}+x_{4}+x_{5}\right)}{x_{1}+x_{4}+x_{5}+2}$.

(3) $\phi\left(x_{1}, x_{2}, x_{3}, x_{4}, x_{5}\right)=\sqrt[3]{x_{1} \cdot x_{4} \cdot x_{5}}$.

Now, we prove our results using these implicit relations.

Theorem 2.1. Let $f$ and $g$ be weakly reciprocally continuous noncompatible self-mappings of a Menger space $(X, F, \Delta)$ satisfying

$$
\begin{gathered}
f(X) \subset g(X) ; \\
\int_{0}^{F(f x, f y, t)} \varphi(s) d s \geq \int_{0}^{F(g x, g y, t)} \varphi(s) d s ; \\
\int_{0}^{F\left(f x, f^{2} x, t\right)} \varphi(s) d s \\
>\int_{0}^{\phi\left(F(g x, g f x, t), F(f x, g x, t), F\left(f^{2} x, g f x, t\right), F(f x, g f x, t), F\left(g x, f^{2} x, t\right)\right)} \varphi(s) d s
\end{gathered}
$$

for all $x, y \in X$ with $f x \neq f^{2} x, t>0$ and $\phi \in \Phi$, where $\varphi: \mathbb{R}^{+} \rightarrow \mathbb{R}$ is a summable non-negative Lebesque integrable mappings such that $\int_{0}^{\epsilon} \varphi(s) d s$ 
for each $\epsilon>0$. If $f$ and $g$ are $R$-weakly commuting of type $\left(A_{g}\right)$ or $R$-weakly commuting of type $\left(A_{f}\right)$ or $R$-weakly commuting of the type $(P)$, then $f$ and $g$ have a common fixed point.

Proof. Since $f$ and $g$ are non-compatible mappings, there exists a sequence $\left\{x_{n}\right\}$ in $X$ such that $\lim _{n \rightarrow \infty} f x_{n}=\lim _{n \rightarrow \infty} g x_{n}=z$ for some $z \in X$, but either $\lim _{n \rightarrow \infty} F\left(f g x_{n}, g f x_{n}, t\right) \neq 1$ or the limit does not exist. Since $f(X) \subset g(X)$, for each $\left\{x_{n}\right\}$, there exists $\left\{y_{n}\right\}$ in $X$ such that $f x_{n}=g y_{n}$. Thus $\lim _{n \rightarrow \infty} f x_{n}=$ $\lim _{n \rightarrow \infty} g x_{n}=\lim _{n \rightarrow \infty} g y_{n}=z$. By virtue of this and using $(C 2)$ we obtain

$$
\int_{0}^{F\left(f x_{n}, f y_{n}, t\right)} \varphi(s) d s \geq \int_{0}^{F\left(g x_{n}, g y_{n}, t\right)} \varphi(s) d s .
$$

Taking $n \rightarrow \infty$, we get

$$
\int_{0}^{F\left(z, \lim _{n \rightarrow \infty} f y_{n}, t\right)} \varphi(s) d s \geq \int_{0}^{F(z, z, t)} \varphi(s) d s=\int_{0}^{1} \varphi(s) d s,
$$

which implies that $F\left(z, \lim _{n \rightarrow \infty} f y_{n}, t\right) \geq 1$ and hence $\lim _{n \rightarrow \infty} f y_{n}=z$. Therefore, we have $\lim _{n \rightarrow \infty} f x_{n}=\lim _{n \rightarrow \infty} g x_{n}=\lim _{n \rightarrow \infty} g y_{n}=z=\lim _{n \rightarrow \infty} f y_{n}$.

Suppose that $f$ and $g$ are $R$-weakly commuting of type $\left(A_{g}\right)$. Then by weak reciprocal continuity of $f$ and $g$, we have $\lim _{n \rightarrow \infty} f g x_{n}=f z$ or $\lim _{n \rightarrow \infty} g f x_{n}=$ $f z$

Let $\lim _{n \rightarrow \infty} g f x_{n}=f z$. Then by $R$-weak commutativity of the type $\left(A_{g}\right)$ of $f$ and $g$, we obtain $F\left(f f y_{n}, g f y_{n}, t\right) \geq F\left(f y_{n}, g y_{n}, t / R\right)$ and hence $\lim _{n \rightarrow \infty} F\left(f f y_{n}, g z, t\right) \geq F(z, z, t / R)=1$, which implies that $\lim _{n \rightarrow \infty} f f y_{n}=$ gz. Also, using $(C 2)$, we get

$$
\int_{0}^{F\left(f f y_{n}, f z, t\right)} \varphi(s) d s \geq \int_{0}^{F\left(g f y_{n}, g z, t\right)} \varphi(s) d s .
$$

Taking $n \rightarrow \infty$, we obtains

$$
\int_{0}^{F(g z, f z, t)} \varphi(s) d s \geq \int_{0}^{F(g z, g z, t)} \varphi(s) d s .
$$

Then we have $f z=g z$. Again by using $R$-weak commutativity of type $\left(A_{g}\right)$, $F(f f z, g f z, t) \geq F(f z, g z, t / R)=1$. This yields $f f z=g f z$ and $f f z=f g z=$ $g f z=g g z$. 
If $f z \neq f f z$, then by using $(C 3)$, we get

$$
\begin{aligned}
& \int_{0}^{F\left(f z, f^{2} z, t\right)} \varphi(s) d s \\
& >\int_{0}^{\phi\left(F(g z, g f z, t), F(f z, g z, t), F\left(f^{2} z, g f z, t\right), F(f z, g f z, t), F\left(g z, f^{2} z, t\right)\right)} \varphi(s) d s \\
& =\int_{0}^{\phi\left(F\left(f z, f^{2} z, t\right), 1,1, F\left(f z, f^{2} z, t\right), F\left(f z, f^{2} z, t\right)\right)} \varphi(s) d s \\
& =\int_{0}^{F\left(f z, f^{2} z, t\right)} \varphi(s) d s,
\end{aligned}
$$

which is a contradiction. Hence $f z=f f z=g f z$ and $f z$ is a common fixed point of $f$ and $g$.

Similarly, if $\lim _{n \rightarrow \infty} f g x_{n}=f z$, then $f z$ is a common fixed point of $f$ and $g$.

Also, if $f$ and $g$ are $R$-weakly commuting of types $\left(A_{f}\right)$ or $(P)$, then the conclusions hold. This completes the proof.

The following corollaries follow easily.

Corollary 2.2. Let $f$ and $g$ be weakly reciprocally continuous noncompatible self-mappings of a Menger space $(X, F, \Delta)$ satisfying the conditions $(C 1),(C 2)$ and

$$
\begin{aligned}
& \int_{0}^{F\left(f x, f^{2} x, t\right)} \varphi(s) d s \\
& >\int_{0}^{\min \left\{F(g x, g f x, t), F(f x, g x, t), F\left(f^{2} x, g f x, t\right), F(f x, g f x, t), F\left(g x, f^{2} x, t\right)\right\}} \varphi(s) d s
\end{aligned}
$$

for all $x, y \in X$ with $f x \neq f^{2} x, t>0$ and $\phi \in \Phi$, where $\varphi: \mathbb{R}^{+} \rightarrow \mathbb{R}$ is a a summable non-negative Lebesque integrable mappings such that $\int_{0}^{\epsilon} \varphi(s) d s$ for each $\epsilon>0$. If $f$ and $g$ are $R$-weakly commuting of type $\left(A_{g}\right)$ or $R$-weakly commuting of type $\left(A_{f}\right)$ or $R$-weakly commuting of the type $(P)$, then $f$ and $g$ have a common fixed point.

Corollary 2.3. Let $f$ and $g$ be weakly reciprocally continuous noncompatible self-mappings of a Menger space $(X, F, \Delta)$ satisfying the conditions 
$(C 1),(C 2)$ and

$$
\begin{aligned}
& \int_{0}^{F\left(f x, f^{2} x, t\right)} \varphi(s) d s \\
& >\int_{0}^{\frac{F(g x, g f x, t)\left(F(g x, g f x, t)+F(f x, g x, t)+F\left(f^{2} x, g f x, t\right)+F(f x, g f x, t)+F\left(g x, f^{2} x, t\right)\right)}{F(g x, g f x, t)+F(f x, g f x, t)+F\left(g x, f^{2} x, t\right)+2}} \varphi(s) d s
\end{aligned}
$$

for all $x, y \in X$ with $f x \neq f^{2} x, t>0$ and $\phi \in \Phi$, where $\varphi: \mathbb{R}^{+} \rightarrow \mathbb{R}$ is a a summable non-negative Lebesque integrable mappings such that $\int_{0}^{\epsilon} \varphi(s) d s$ for each $\epsilon>0$. If $f$ and $g$ are $R$-weakly commuting of type $\left(A_{g}\right)$ or $R$-weakly commuting of type $\left(A_{f}\right)$ or $R$-weakly commuting of the type $(P)$, then $f$ and $g$ have a common fixed point.

Corollary 2.4. Let $f$ and $g$ be weakly reciprocally continuous noncompatible self-mappings of a Menger space $(X, F, \Delta)$ satisfying the conditions $(C 1),(C 2)$ and

$$
\int_{0}^{F\left(f x, f^{2} x, t\right)} \varphi(s) d s>\int_{0}^{\sqrt[3]{F(g x, g f x, t) \cdot F(f x, g f x, t) \cdot F\left(g x, f^{2} x, t\right)}} \varphi(s) d s
$$

for all $x, y \in X$ with $f x \neq f^{2} x, t>0$ and $\phi \in \Phi$, where $\varphi: \mathbb{R}^{+} \rightarrow \mathbb{R}$ is a a summable non-negative Lebesque integrable mappings such that $\int_{0}^{\epsilon} \varphi(s) d s$ for each $\epsilon>0$. If $f$ and $g$ are $R$-weakly commuting of type $\left(A_{g}\right)$ or $R$-weakly commuting of type $\left(A_{f}\right)$ or $R$-weakly commuting of the type $(P)$, then $f$ and $g$ have a common fixed point.

The Implict Relation $(\Psi)$ : Let $\Psi$ denote the family of functions $\psi$ : $[0,1]^{4} \rightarrow[0,1]$ such that $\psi$ is continuous and $\psi(x, 1, x, 1)=x$.

These are examples.

(1) $\psi_{1}\left(x_{1}, x_{2}, x_{3}, x_{4}\right)=\min \left\{x_{1}, x_{2}, x_{3}, x_{4}\right\}$.

(2) $\psi_{2}\left(x_{1}, x_{2}, x_{3}, x_{4}\right)=\sqrt{x_{1} \cdot x_{3}}$.

Next, we prove our main results.

Theorem 2.5. Let $f$ and $g$ be weakly reciprocally continuous noncompatible self-mappings of a Menger space $(X, F, \Delta)$ satisfying the conditions 
$(C 1),(C 2)$ and

$$
\begin{aligned}
& \int_{0}^{F\left(f x, f^{2} x, t\right)} \varphi(s) d s \\
& >\int_{0}^{\psi\left(F(g x, g f x, t), F(f x, g x, t), F(f x, g f x, t), F\left(f^{2} x, g f x, t\right)\right)} \varphi(s) d s
\end{aligned}
$$

for all $x, y \in X$ with $f x \neq f^{2} x, t>0$ and $\psi \in \Psi$, where $\varphi: \mathbb{R}^{+} \rightarrow \mathbb{R}$ is a a summable non-negative Lebesque integrable mappings such that $\int_{0}^{\epsilon} \varphi(s) d s$ for each $\epsilon>0$. If $f$ and $g$ are $R$-weakly commuting of type $\left(A_{g}\right)$ or $R$-weakly commuting of type $\left(A_{f}\right)$ or $R$-weakly commuting of the type $(P)$, then $f$ and $g$ have a common fixed point.

Proof. As in the proof of Theorem 2.1, we obtain $f f z=g f z$ and $f f z=$ $f g z=g f z=g g z$.

If $f z \neq f f z$, then by using $(C 7)$, we get

$$
\begin{aligned}
& \int_{0}^{F\left(f z, f^{2} z, t\right)} \varphi(s) d s \\
& >\int_{0}^{\psi\left(F(g z, g f z, t), F(f z, g z, t), F(f z, g f z, t), F\left(f^{2} z, g f z, t\right)\right)} \varphi(s) d s \\
& =\int_{0}^{\psi\left(F\left(f z, f^{2} z, t\right), 1, F\left(f z, f^{2} z, t\right), 1\right)} \varphi(s) d s \\
& =\int_{0}^{F\left(f z, f^{2} z, t\right)} \varphi(s) d s,
\end{aligned}
$$

which is a contradiction. Hence $f z=f f z=g f z$ and $f z$ is a common fixed point of $f$ and $g$.

Similarly, if $\lim _{n \rightarrow \infty} f g x_{n}=f z$, then $f z$ is a common fixed point of $f$ and $g$.

Also, if $f$ and $g$ are $R$-weakly commuting of types $\left(A_{f}\right)$ or $(P)$, then the conclusions hold. This completes the proof.

The following corollaries follow easily.

Corollary 2.6. Let $f$ and $g$ be weakly reciprocally continuous noncompatible self-mappings of a Menger space $(X, F, \Delta)$ satisfying the conditions 
$(C 1),(C 2)$ and

$$
\begin{aligned}
& \int_{0}^{F\left(f x, f^{2} x, t\right)} \varphi(s) d s \\
& >\int_{0}^{\min \left\{F(g x, g f x, t), F(f x, g x, t), F(f x, g f x, t), F\left(f^{2} x, g f x, t\right)\right\}} \varphi(s) d s
\end{aligned}
$$

for all $x, y \in X$ with $f x \neq f^{2} x, t>0$ and $\psi \in \Psi$, where $\varphi: \mathbb{R}^{+} \rightarrow \mathbb{R}$ is a a summable non-negative Lebesque integrable mappings such that $\int_{0}^{\epsilon} \varphi(s) d s$ for each $\epsilon>0$. If $f$ and $g$ are $R$-weakly commuting of type $\left(A_{g}\right)$ or $R$-weakly commuting of type $\left(A_{f}\right)$ or $R$-weakly commuting of the type $(P)$, then $f$ and $g$ have a common fixed point.

Corollary 2.7. Let $f$ and $g$ be weakly reciprocally continuous noncompatible self-mappings of a Menger space $(X, F, \Delta)$ satisfying the conditions $(C 1),(C 2)$ and

$$
\int_{0}^{F\left(f x, f^{2} x, t\right)} \varphi(s) d s>\int_{0}^{\sqrt{F(g x, g f x, t) \cdot F(f x, g f x, t)}} \varphi(s) d s
$$

for all $x, y \in X$ with $f x \neq f^{2} x, t>0$ and $\psi \in \Psi$, where $\varphi: \mathbb{R}^{+} \rightarrow \mathbb{R}$ is a a summable non-negative Lebesque integrable mappings such that $\int_{0}^{\epsilon} \varphi(s) d s$ for each $\epsilon>0$. If $f$ and $g$ are $R$-weakly commuting of type $\left(A_{g}\right)$ or $R$-weakly commuting of type $\left(A_{f}\right)$ or $R$-weakly commuting of the type $(P)$, then $f$ and $g$ have a common fixed point.

\section{Acknowledgements}

One of the authors (Professor Sanjay Kumar) would like to acknowledge University Grants Comission for providing financial grant Major Research Project under Ref. 39-41/2010(SR).

\section{References}

[1] M. Imdad, J. Ali, Some common fixed point theorems in fuzzy metric spaces, Math. Commun., 11, No. 2 (2006), 153-163. 
[2] J.K. Kohli, S. Vashistha, Common fixed point theorems in probabilistic metric spaces, Acta Math. Hungar., 115, No. 1-2 (2007), 37-47, doi: 10.1007/s10474-006-0533-7

[3] K. Menger, Statistical metrics, Proc. Natl. Acad. Sci., USA, 28 (1942), 535-537, doi: 10.1073/pnas.28.12.535

[4] S.N. Mishra, Common fixed points of compatible mappings in probabilistic metric spaces, Math. Japon., 36, No. 2 (1991), 283-289.

[5] R.P. Pant, A common fixed point theorem under a new condition, Indian J. Pure Appl. Math., 30, No. 2 (1999), 147-152, 1999.

[6] R.P. Pant, R.K. Bisht and D. Arora, Weak reciprocal continuity and fixed point theorems, Ann. Univ. Ferrara., 57, No. 1 (2011), 181-190, doi: 10.1007/s11565-011-0119-3

[7] H.K. Pathak, Y.J. Cho, S.M. Kang, Remarks on $R$-weakly commuting mappings and common fixed point theorems, Bull. Korean Math. Soc., 34, No. 2 (1997), 247-257.

[8] B. Schweizer, A. Sklar, Probabilistic Metric Spaces, North-Holland Series in Probability and Applied Math., North-Holland Publ. Co., New York, 1983.

[9] V.M. Sehgal, A.T. Bharucha-Reid, Fixed points of contraction mappings in probabilistic metric spaces, Math. Systems Theory, 6 (1972), 97-102, doi: $10.1007 / \mathrm{BF} 01706080$. 
